\title{
Spatiotemporal Representation of the Pitch of Harmonic Complex Tones in the Auditory Nerve
}

\author{
Leonardo Cedolin ${ }^{1,3}$ and Bertrand Delgutte ${ }^{1,2}$ \\ ${ }^{1}$ Eaton-Peabody Laboratory, Massachusetts Eye and Ear Infirmary, Boston, Massachusetts 02114, and ${ }^{2}$ Research Laboratory of Electronics, Massachusetts \\ Institute of Technology, and ${ }^{3}$ Speech and Hearing Bioscience and Technology Program, Harvard-Massachusetts Institute of Technology Division of Health \\ Sciences and Technology, Cambridge, Massachusetts 02139
}

The pitch of harmonic complex tones plays an important role in speech and music perception and the analysis of auditory scenes, yet traditional rate-place and temporal models for pitch processing provide only an incomplete description of the psychophysical data. To test physiologically a model based on spatiotemporal pitch cues created by the cochlear traveling wave (Shamma, 1985), we recorded from single fibers in the auditory nerve of anesthetized cat in response to harmonic complex tones with missing fundamentals and equalamplitude harmonics. We used the principle of scaling invariance in cochlear mechanics to infer the spatiotemporal response pattern to a given stimulus from a series of measurements made in a single fiber as a function of fundamental frequency $F_{0}$. We found that spatiotemporal cues to resolved harmonics are available for $F_{0}$ values between 350 and $1100 \mathrm{~Hz}$ and that these cues are more robust than traditional rate-place cues at high stimulus levels. The lower $F_{0}$ limit is determined by the limited frequency selectivity of the cochlea, whereas the upper limit is caused by the degradation of phase locking to the stimulus fine structure at high frequencies. The spatiotemporal representation is consistent with the upper $F_{0}$ limit to the perception of the pitch of complex tones with a missing fundamental, and its effectiveness does not depend on the relative phase between resolved harmonics. The spatiotemporal representation is thus consistent with key trends in human psychophysics.

\section{Introduction}

Harmonic complex tones present in speech, animal vocalizations, and the sounds of musical instruments produce a strong pitch sensation at their fundamental frequency $F_{0}$. Although the pitch of complex tones plays an important role in music, speech, and auditory scene analysis, the neural mechanisms for pitch perception are still poorly understood. Despite the report of pitchselective neurons in the auditory cortex of a primate (Bendor and Wang, 2005), the computations underlying these cortical responses and the neural codes on which these computations operate are unknown. Studies of the coding of harmonic complex tones in the auditory nerve (AN) and cochlear nucleus have primarily focused on temporal pitch cues available in interspike interval distributions (Javel, 1980; Evans, 1983; Palmer, 1990; Shofner, 1991, 1999; Rhode, 1995; Cariani and Delgutte, 1996a,b; Wiegrebe and Winter, 2001; Winter et al., 2001; Cedolin and Delgutte, 2005; Sayles and Winter, 2008). These cues are closely related to the autocorrelation model of pitch, which accounts for a wide variety of pitch phenomena (Licklider, 1951; Meddis and Hewitt, 1991a,b; de Cheveigné, 2005). Fewer studies have focused

Received Dec. 23, 2009; revised July 28, 2010; accepted July 31, 2010.

This work was supported by National Institutes of Health Grants R01 DC002258 and P30 DC005209. We thank Connie Miller for surgical assistance and Ken Hancock for software support. Bob Carlyon, Ken Hancock, Andrew Oxenham, Shihab Shamma, Chris Shera, Garrett Stanley, and Grace Wang made valuable comments on this manuscript.

Correspondence should be addressed to Bertrand Delgutte, Massachusetts Eye and Ear Infirmary, Eaton-Peabody Laboratory, 243 Charles Street, Boston, MA 02114. E-mail: bertrand_delgutte@meei.harvard.edu.

DOI:10.1523/JNEUROSCI.6365-09.2010

Copyright $\odot 2010$ the authors $\quad 0270-6474 / 10 / 3012712-13 \$ 15.00 / 0$ on "place" or "spectral" cues based on the cochlear frequency map and the mechanical frequency analysis of individual harmonics in the cochlea (Sachs and Young, 1979; Cedolin and Delgutte, 2005). Such place cues provide an appropriate input to harmonic template models for pitch extraction (Goldstein, 1973; Wightman, 1973; Cohen et al., 1995). Although either rate-place or interspike interval information from AN fibers in cat supports precise pitch estimation over the $F_{0}$ range of cat vocalizations (500-1000 $\mathrm{Hz}$ ), neither representation is entirely consistent with human psychophysical data (Cedolin and Delgutte, 2005). The rateplace representation degrades with increasing sound level and also fails to predict the existence of an upper frequency limit for pitch (Schouten et al., 1962; Moore, 1973). The interval representation does not account for the greater salience of pitch based on resolved harmonics compared with pitch based on unresolved harmonics (Carlyon and Shackleton, 1994; Carlyon, 1998). Here, we investigate an alternative, spatiotemporal representation of pitch (Shamma, 1985) aimed at combining the advantages and overcoming the limitations of traditional representations.

Sinusoidal stimulation of the cochlea gives rise to a traveling wave that moves from base to apex, progressively slowing down as it approaches the cochlear location tuned to the stimulus frequency, where the phase of basilar membrane velocity changes rapidly (Robles and Ruggero, 2001). At frequencies within the range of phase locking, this rapid phase transition is reflected in the timing of AN spike discharges (Anderson et al., 1971; Pfeiffer and Kim, 1975; van der Heijden and Joris, 2006; Palmer and Shackleton, 2009; Temchin and Ruggero, 2010). For harmonic 


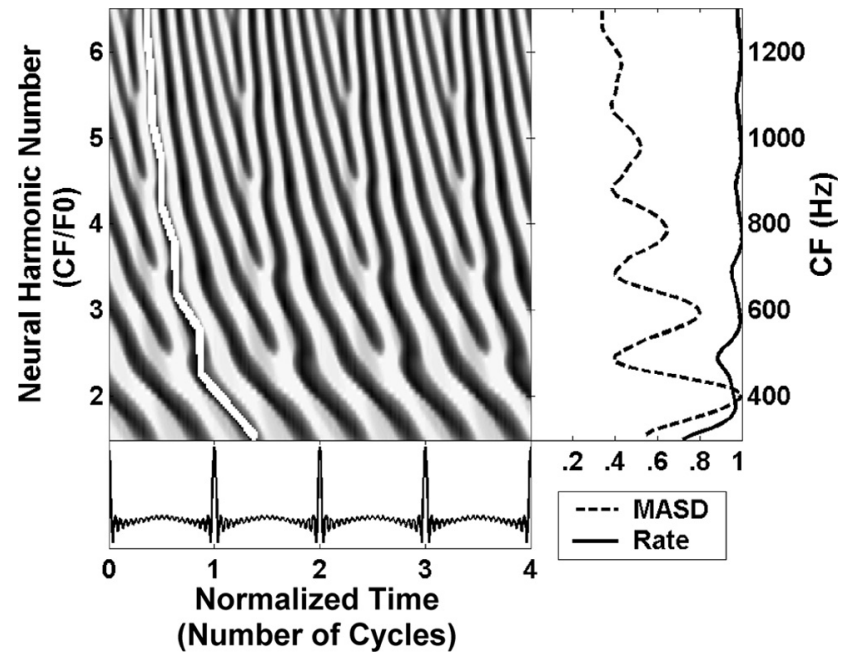

Figure 1. Spatiotemporal activity pattern of the human peripheral auditory model of Zhang et al. (2001) in response to a harmonic complex tone with $F_{0}$ of $200 \mathrm{~Hz}$ at $50 \mathrm{~dB}$ SPL. Left, The model response is displayed as a function of time (in dimensionless units, $t \times F_{0}$ ) and cochlear place, which maps to $C F$, expressed as the dimensionless ratio $C F / F_{0}$ (neural harmonic number). Fast variations in response latency with $C F$ at integer neural harmonic numbers are highlighted by the white line. The right panel shows the spatial profiles of average rate (solid line) and MASD (dashed), derived from the spatiotemporal pattern on the left. The rate and MASD profiles are normalized by their respective maximum value.

complex tones, a rapid phase transition is expected to occur at the spatial locations tuned to each resolved harmonic (see Fig. 1). These spatiotemporal cues to resolved harmonics could be extracted by a neural mechanism sensitive to the relative timing of spikes from adjacent cochlear locations (Shamma, 1985; Carney, 1990).

We tested the spatiotemporal representation of pitch by recording the responses of AN fibers in anesthetized cats to harmonic complex tones with $F_{0}$ varied in fine increments. We find that this representation is more robust to variations in stimulus level than the rate-place representation and also predicts an upper frequency limit to pitch consistent with psychophysical data.

\section{Materials and Methods}

Spatiotemporal pitch cues in a peripheral auditory model. The spatiotemporal representation of pitch is based on phase transition cues to the frequencies of resolved harmonics created by the cochlear traveling wave. Figure 1 shows the spatiotemporal pattern of AN activity produced by a physiologically realistic peripheral auditory model (Zhang et al., 2001) in response to a harmonic complex tone with missing fundamental at 200 $\mathrm{Hz}$. The response pattern is shown as a function of both time (expressed in dimensionless units $t \times F_{0}$ ) and position along the cochlea, which maps to characteristic frequency $(\mathrm{CF})$. The $\mathrm{CF}$ is expressed by the dimensionless ratio $\mathrm{CF} / F_{0}$, which we call "neural harmonic number." In this example, a neural harmonic number of 3 corresponds to a CF of $600 \mathrm{~Hz}$. As predicted, the latency of the traveling wave changes more rapidly with $\mathrm{CF}$ at cochlear locations tuned to low-order harmonics than for CFs in between two harmonics (Fig. 1, white line). To extract these cues, we use a spatial derivative operation that simulates a hypothetical lateral inhibitory mechanism operating on the spatiotemporal pattern of AN activity (Shamma, 1985). Specifically, we compute the point-by-point difference between adjacent rows in Figure 1, and then integrate the absolute value of the difference over time. The resulting "mean absolute spatial derivative" (MASD) shows local maxima at CFs corresponding to the frequencies of harmonics $2-6$, whereas the average firing rate is mostly saturated at this stimulus level [ $50 \mathrm{~dB}$ sound pressure level (SPL) per component]. Thus, the model predicts that spatiotemporal pitch cues persist at stimulus levels where the rate-place representation is degraded.

The most direct way to study the spatiotemporal representation of pitch would be to measure the AN response to a given harmonic complex tone as a function of both time and CF. Since a fine, regular sampling of the tonotopic axis is hard to achieve with single-unit recordings, we relied instead on the principle of scaling invariance in cochlear mechanics (Zweig, 1976) to infer the spatiotemporal response pattern from measurements made at a single cochlear location. In a cochlea with perfect scaling invariance, the response to a pure tone of frequency $f$ at the cochlear location tuned to CF depends only on the ratio f/CF (Zweig, 1976). This means that the magnitude and phase of the cochlear response to a pure tone of frequency $f_{d}$ at the location tuned to $\mathrm{CF}_{d}$ are equal to the magnitude and the phase of the response of the cochlear location $\mathrm{CF}_{p}$ to a tone of frequency $f_{p}=\mathrm{CF}_{p} f_{d} / \mathrm{CF}_{d}$. Thus, to obtain the spatial pattern of response to a tone of desired frequency $f_{d}$ for the set of cochlear locations tuned to $\left\{\mathrm{CF}_{d}\right\}$, it suffices, in principle, to measure responses at one location tuned to $\mathrm{CF}_{p}$ to the set of probe frequencies $\left\{f_{p}\right\}=\mathrm{CF}_{p} f_{d} /\left\{\mathrm{CF}_{d}\right\}$. The same reasoning applies to a harmonic complex tone in which all the components are multiples of a common fundamental. Thus, the spatiotemporal response pattern to a complex tone with a given $F_{0}$ can, in principle, be inferred from the responses recoded in a single AN fiber to a series of complex tones with varying $F_{0}$.

Figure 2 illustrates the scaling invariance principle using the model of Zhang et al. (2001) for peripheral auditory processing in cat. The left panel shows the model spatiotemporal response pattern to a harmonic complex tone with fixed $F_{0}(500 \mathrm{~Hz})$ for CFs ranging from 750 to 2250 $\mathrm{Hz}$. The right panel shows the model temporal response patterns at a single cochlear place $\left(\mathrm{CF}_{0}, 1500 \mathrm{~Hz}\right)$ to a series of complex tones with $F_{0}$ values varying from 333 to $1000 \mathrm{~Hz}$. The $F_{0}$ values and CFs were chosen so that the neural harmonic number $\mathrm{CF} / F_{0}$ ranges from 1.5 to 4.5 in both panels. In addition, the time axis is expressed in dimensionless units $\tau=$ $t \times F_{0}$ (number of stimulus cycles). The spatiotemporal response patterns for the two conditions are nearly indistinguishable: they both show fast latency changes around integer values of neural harmonic number $(2,3,4)$ and relatively more constant latencies around noninteger harmonic numbers $(2.5,3.5)$. Average discharge rate and MASD, computed as in Figure 1, also exhibit nearly identical features in the two panels, with peaks at integer values of neural harmonic number and valleys in between. Thus, these model simulations support the use of scaling invariance to infer the response of a population of AN fibers to a given complex tone by measuring the response of a single AN fiber to a series of complex tones with varying $F_{0}$.

Scaling invariance is a good approximation when applied to a local region of the cochlea but does not hold over wide cochlear spans (Shera and Guinan, 2003; van der Heijden and Joris, 2006). Since, for each fiber, $F_{0}$ was varied over a limited range in our experiments ( $\sim 1.6$ octave), deviations from scaling invariance may not present a major problem, as Figure 2 suggests. This point is addressed further in Discussion.

Neurophysiological procedure. Methods for recording from auditorynerve fibers in anesthetized cats were as described by Cedolin and Delgutte (2005) and were approved by the Animal Care and Use Committees of both the Massachusetts Eye and Ear Infirmary and the Massachusetts Institute of Technology. Cats were anesthetized with Dial in urethane $(75 \mathrm{mg} / \mathrm{kg})$, with supplementary doses given as needed to maintain an areflexic state. The posterior fossa was opened and the cerebellum retracted to expose the auditory nerve. The tympanic bullae were opened to expose the round window. Throughout the experiment, the cat was given injections of dexamethasone $(0.26 \mathrm{mg} / \mathrm{kg}$, i.m. every $4 \mathrm{~h})$ to prevent brain swelling, and Ringer's solution $(50 \mathrm{ml} / \mathrm{d}$, i.v.) to prevent dehydration. General physiological state was assessed by monitoring heart rate, respiratory rate, exhaled $\mathrm{CO}_{2}$ concentration, and rectal temperature, which was maintained at $37^{\circ} \mathrm{C}$ by a thermostat-controlled heating pad.

The cat was placed on a vibration-isolated table in an electrically shielded, soundproof chamber. A silver electrode positioned near the round window was used to measure the compound action potential of the $\mathrm{AN}$ in response to click stimuli, to assess the condition and stability of cochlear function.

Sound was delivered to the cat's ear through a calibrated closed acoustic assembly driven by an electrodynamic speaker (Realistic 40-1377). Stimuli were generated by a 16-bit digital-to-analog converter (National Instruments NIDAC 6052E) using sampling rates of 20 or $50 \mathrm{kHz}$. Stim- 
uli were digitally filtered to compensate for the transfer characteristics of the acoustic system.

Spikes were recorded with glass micropipettes filled with $2 \mathrm{~m} \mathrm{KCl}$. The electrode was inserted into the nerve and then mechanically advanced using a micropositioner (Kopf 650). The electrode signal was bandpass filtered $(0.3-3 \mathrm{kHz})$ and fed to a custom spike detector. The times of spike peaks were recorded with 1 $\mu$ s resolution and saved to disk for subsequent analysis.

A click at $\sim 60 \mathrm{~dB}$ SPL was used as search stimulus. On isolation of a single unit, a frequency tuning curve was measured by an automatic tracking algorithm (Kiang and Moxon, 1974) using $50 \mathrm{~ms}$ tone bursts to determine the $\mathrm{CF}$. The spontaneous firing rate (SR) of the fiber was measured over $20 \mathrm{~s}$. The responses to complex-tone stimuli were then studied.

Stimuli. Stimuli were series of harmonic complex tones consisting of 19 equalamplitude harmonics [numbers 2-20 (i.e., excluding the fundamental)]. The fundamental frequency $\left(F_{0}\right)$ was stepped up and down such that the ratio of the fiber CF to $F_{0}$ (the neural harmonic number) typically varied from 1.5 to 4.5 in increments of $\pm 1 / 8$. This $F_{0}$ variation causes harmonics $2-4$ (which are important for determining the pitch of missing fundamental stimuli) to successively traverse the auditory filter centered at the CF. If these harmonics are resolved by the cochlea, there should be a regular modulation in both firing rate and response latency as $F_{0}$ varies. Each complex-tone series consisted of 25 ascending $F_{0}$ steps and 25 descending steps, each lasting $200 \mathrm{~ms}$, including a $20 \mathrm{~ms}$ transition during which the waveform for one $F_{0}$ gradually decayed while overlapping with the gradual buildup of the waveform for the subsequent $F_{0}$. Responses were typically collected over 20 repetitions of the $10 \mathrm{~s}$ stimulus with no interruption.

The sound pressure level of each harmonic was initially set at $10-15 \mathrm{~dB}$ above the threshold of the fiber for a pure tone at CF. When possible, the stimulus level was then varied over a $20-30 \mathrm{~dB}$ range to investigate the robustness of the spatiotemporal representation. All stimulus levels in this paper refer to the sound pressure of one frequency component of the complex tone. Since the stimuli contain 19 equal-amplitude components, the overall sound pressure level is $12.8 \mathrm{~dB}$ higher.

Psychophysical studies show that the pitch and pitch strength of harmonic complex tones containing resolved harmonics are mostly independent of the phase relationships among harmonics (Houtsma and Smurzynski, 1990; Carlyon and Shackleton, 1994; Bernstein and Oxenham, 2005). To evaluate the robustness of the spatiotemporal representation to the phase pattern, three versions of each stimulus were generated with different phase relationships among the harmonics: cosine phase, alternating sine-cosine phase, and negative "Schroeder" phase (Schroeder, 1970). The three stimuli have the same power spectrum and autocorrelation function, but sharply differ in their temporal envelopes (see Fig. 9). The cosine-phase stimulus has a very "peaky" envelope periodic at $F_{0}$. The envelope of the alternating-phase stimulus is also very peaky, but its periodicity is at $2 \times F_{0}$, even though the periodicity of the waveform remains at $F_{0}$. Finally, a Schroeder phase relationship among the harmonics ensures the envelope is nearly flat.

Data analysis. The first step in the analysis was to select the spikes occurring during the $180 \mathrm{~ms}$ steady-state portion of each $F_{0}$ step, excluding the $20 \mathrm{~ms}$ transition period between steps. The nonscaling conduction delay for each cochlear location $\left[T_{d}\right.$ parameter in the study by Carney and Yin (1988)] was subtracted from the time of each spike. Period histograms were computed using 50 bins per cycle of $F_{0}$ and displayed as a function of both time and $F_{0}$, as in Figure 3. Period histograms obtained with the same $F_{0}$ during the ascending and descending parts of the $F_{0}$ sequence were added together. To apply scaling invariance, the time and $F_{0}$ axes were expressed in dimensionless units $\tau=t \times F_{0}$ (number of stimulus cycles) and $n=\mathrm{CF} / F_{0}$ (neural harmonic number), respectively (see Fig. 3, left panels). Two metrics were computed from the resulting pseudo-spatiotemporal response pattern: the average discharge rate, obtained by integrating each period histogram over time and converting to spikes/second, and the MASD, obtained by differentiating the pseudo-spatiotemporal pattern with respect to neural harmonic number, and then taking the absolute value and integrating over time.

Both the average rate and the MASD were plotted against neural harmonic number after smoothing by a three-point triangular filter with weights $[1 / 4,1 / 2,1 / 4]$ (see Fig. 3, right panels). Integer values of neural harmonic number occur when the fiber CF coincides with one of the harmonics of the stimulus, whereas the neural harmonic number is an odd integer multiple of $0.5(2.5,3.5,4.5)$ when the CF falls halfway between two harmonics. Resolved harmonics are expected to result in peaks in either rate or MASD (or both) near integer values of the neural harmonic number.

We used "bootstrap" resampling (Efron and Tibshirani, 1993) of the spike trains recorded from each fiber to evaluate the statistical reliability of the estimates of average rate and MASD. Specifically, 100 resampled data sets were generated by drawing with replacement from the set of spike trains, typically recorded over 20 stimulus repetitions. For each $F_{0}$, spike trains in response to the ascending and descending part of the $F_{0}$ sequence were drawn independently from each other. The average discharge rate and MASD were computed from each bootstrap data set, and the SDs of these measures were used to obtain error bars on the estimates.

To quantitatively describe the data from each fiber, a simple mathematical function was fit separately to profiles of average rate and MASD against neural harmonic number $n$ as follows:

$$
f(n)=A \cos (2 \pi \varphi n) e^{\frac{-n}{n_{0}}}+B e^{\frac{-n}{n_{0}}}+C .
$$

This expression is the sum of an exponentially decaying sinusoid in cosine phase, a constant term $C$, and an exponential term. $\varphi$ represents the normalized frequency of the oscillating component relative to the CF; the sinusoid peaks exactly at integer values of the neural harmonic number when $\varphi=1$. The function has five free parameters: the amplitude $A$ and normalized frequency $\varphi$ of the damped oscillation, the decay parameter $n_{0}$ of the exponentials (constrained to be the same for the baseline and oscillatory components), the amplitude $B$ of the exponential term, and the constant term $C$. Equation 1 was fit to the data by the least-squares method using the Levenberg-Marquardt algorithm as implemented by Matlab's "lsqcurvefit" function. Figure $5 A$ shows example fits to rate and MASD profiles for one AN fiber. 


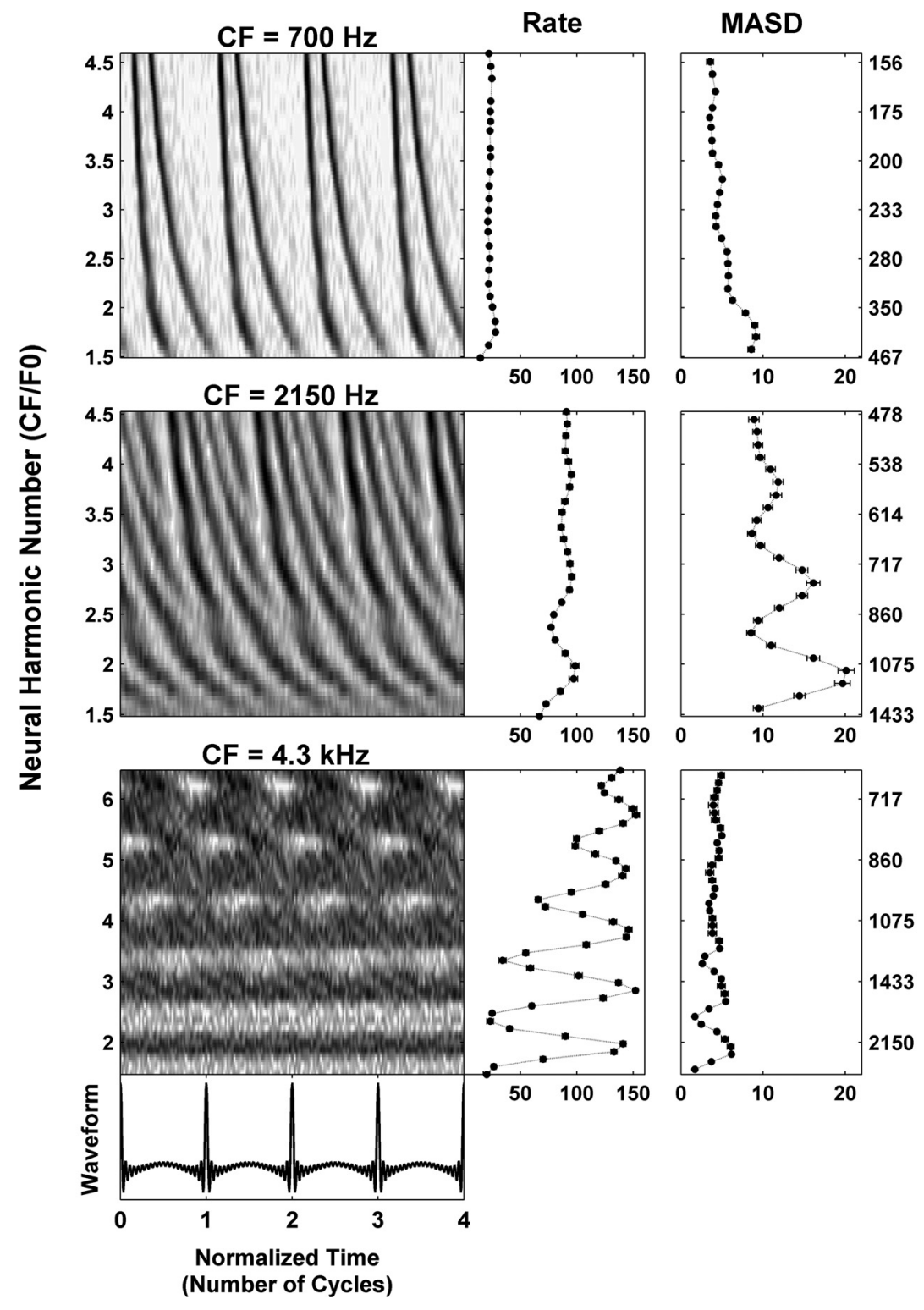

Figure 3. Responses of three AN fibers with (Fs of $700 \mathrm{~Hz}$ (top), $2150 \mathrm{~Hz}$ (middle), and $4300 \mathrm{~Hz}$ (bottom) to series of harmonic complex tones in cosine phase. Left panels, Pseudo-spatiotemporal discharge pattern, displayed as a function of normalized time (horizontal axis) and neural harmonic number $\left(F / F_{0}\right.$ (vertical axis). Right panels, Firing rate and MASD derived from the corresponding pseudo-spatiotemporal response patterns on the left.

To assess the reliability of the rate-place and spatiotemporal cues to resolved harmonics, we compared the ability of the five-parameter model (Equation 1) to fit each rate-place or MASD profile with that of a threeparameter model lacking an oscillatory component (i.e., with $A$ set to 0 in Equation 1). The pitch cues were only considered reliable if an $F$ test for the ratio of variances of the residuals indicated a significantly better fit $(p<0.01)$ for the model with an oscillatory component.

To quantitatively characterize how well resolved harmonics are represented in rate and MASD profiles, we used the oscillatory fitted curves to compute metrics such as the "harmonic strength" (see Fig. 6) and the best frequency for complex tones, the product $\varphi \times$ CF (see Fig. 8). Specifically, we fit the model to 100 bootstrap resamplings of the rate and MASD data for each $F_{0}$ series and computed the metrics for each bootstrap resampling. In Figures 6-8, we report the median values of these metrics across all bootstrap resamplings. These median values showed less variability across fibers compared with estimates based on the original data set.

\section{Results}

Our results are based on responses to 240 harmonic complex-tone series recorded from 102 auditory-nerve fibers in five cats. Of these, 72 fibers (71\%) had high SR (>18 spikes/s) (Liberman 1978), 4 had low SR ( $<0.5$ spike/s), and $26(25 \%)$ had medium SR. The CFs of the fibers ranged from 300 to $5900 \mathrm{~Hz}$, with $55 \%$ between 1 and $3 \mathrm{kHz}$. We focused on fibers with CFs $<5-6 \mathrm{kHz}$ because phase locking to the waveform fine time structure is critical for the spatiotemporal representation. Stimulus levels ranged from 5 to $85 \mathrm{~dB}$ SPL per component, with $80 \%$ of the data between 25 and $65 \mathrm{~dB}$ SPL.

\section{Spatiotemporal pitch cues in single-fiber responses}

Figure 3 shows the responses to complextone series with harmonics in cosine phase for three auditory-nerve fibers with CFs of $700 \mathrm{~Hz}$ (A), $2150 \mathrm{~Hz}$ (B), and 4300 $\mathrm{Hz}(\mathrm{C})$, respectively. The left panels show pseudo-spatiotemporal patterns (based on period histograms) as a function of both normalized time $\left(t \times F_{0}\right.$; horizontal axis) and neural harmonic number $\left(\mathrm{CF} / F_{0}\right.$; verti$\mathrm{cal}$ axis). The right panels show the average discharge rate and the MASD obtained from the pseudo-spatiotemporal response patterns on the left (see Materials and Methods) as a function of neural harmonic number. An additional vertical axis on the right shows the stimulus $F_{0}$ values corresponding to the neural harmonic numbers on the left axis.

For the low-CF fiber (Fig. 3, top), the response latency decreases monotonically with increasing neural harmonic number. This smooth variation is reflected in the absence of peaks at integer neural harmonic numbers in either rate or MASD. Thus, at this low CF, neither the rateplace nor the spatiotemporal representation provides evidence for resolved harmonics for $F_{0}$ values in the range tested $(156-467 \mathrm{~Hz})$. This is consistent with a previous report that rate-place cues to resolved harmonics are rarely observed for $F_{0}$ values $<400-500 \mathrm{~Hz}$ in cat because of the limited cochlear frequency resolution at low CFs (Cedolin and Delgutte, 2005).

The pseudo-spatiotemporal response pattern of the fiber with CF at $2150 \mathrm{~Hz}$ (Fig. 3, middle) does show staircase-like variations in response latency with neural harmonic number that are qualitatively similar to those predicted by the peripheral auditory model in Figure 2. Specifically, the response latency varies rapidly with neural harmonic number near integer neural harmonic numbers, whereas it changes more slowly at neural harmonic numbers that are halfway between integers. As a result, the MASD shows local maxima near neural harmonic numbers 2, 3, and 4, thus providing evidence for spatiotemporal pitch cues in the AN response. The mean firing rate also shows peaks at integer neural 
harmonic numbers, although they are less pronounced than those of the MASD at this moderate stimulus level $[20 \mathrm{~dB}$ relative to (re.) threshold].

The pseudo-spatiotemporal response pattern of the $4.3 \mathrm{kHz}$ fiber (Fig. 3, bottom) shows dark horizontal bands representing increased activity at integer values of the neural harmonic number. These bands are reflected in the rate-place profile, where harmonics 2-6 are resolved despite the relatively high stimulus level (30 $\mathrm{dB}$ re. threshold). This strong rate-place coding is consistent with the progressive sharpening of the bandwidths of cochlear filters relative to their center frequency with increasing CF (Kiang et al., 1965; Shera et al., 2002; Cedolin and Delgutte, 2005). In contrast, no obvious latency cues are apparent in the pseudo-spatiotemporal response pattern, and the MASD only shows small peaks at neural harmonic numbers 2 and 3, which likely reflect increased Poisson-like noise when the firing rate is high rather than genuine spatiotemporal cues. This degradation in spatiotemporal cues at higher CFs is consistent with the steep decline in phase locking to the fine time structure $>3 \mathrm{kHz}$ in the cat AN (Johnson, 1980).

We have hypothesized that the spatiotemporal representation of pitch may be effective at higher stimulus levels where the rate-place representation breaks down because of saturation of the rate-level functions. Figure 4 shows the responses of an AN fiber (CF, $1920 \mathrm{~Hz}$ ) to a series of harmonic complex tones at 10,25 , and 40 $\mathrm{dB}$ above the fiber's threshold at CF (25 $\mathrm{dB}$ SPL). At the lower level (top), the second, third, and possibly fourth harmonic appear as distinct peaks in both rate and MASD profiles. As the level of each harmonic is increased to $25 \mathrm{~dB}$ above threshold (middle), the rate begins to saturate so that only the second harmonic is convincingly resolved. In contrast, strong latency cues to the second, third, and possibly fourth harmonic are still present in the pseudo-spatiotemporal response pattern, resulting in corresponding peaks in the MASD. At the highest level tested ( $40 \mathrm{~dB}$ re. threshold, bottom) the rate is almost completely saturated, whereas peaks at the second and third harmonic are still detectable in the MASD. This example supports our hypothesis that a spatiotemporal representation of pitch might still work at stimulus levels for which a strictly rate-based representation is severely degraded.

\section{Spatiotemporal representation of pitch by the AN fiber population}

To quantitatively compare the strengths of the pitch cues provided by the rate-place and spatiotemporal representations, a mathematical function was fit independently to the profiles of average rate and MASD against neural harmonic number for each set of measured responses (see Materials and Methods). An example is shown in Figure $5 A$ for the same fiber as in the middle panels of Figure 3 (CF, $2150 \mathrm{~Hz}$ ). The fitted curves (solid lines) closely capture the oscillations in both rate and MASD profiles. The more pronounced these oscillations, the better individual harmonics are resolved. In Figure $5 A$, the oscillations for the MASD seem more prominent than those for the rate. We use the area between the top and bottom envelopes of the fitted curve (Fig. $5 A$, light shadings) to characterize the strength of the oscillations in the rate and MASD profiles. Since the two metrics (rate and MASD) have different values (and units), the oscillation area was normalized by the median SD of the data points (Fig. $5 \mathrm{~A}$, dark shadings) to allow direct comparisons between the two representations. The SDs are obtained by bootstrap resampling of the spike trains across stimulus presentations (see Materials and Methods) and we use the median SD across all $F_{0}$ values tested. The resulting metric, which we call harmonic strength, is analogous to the sensitivity index $d^{\prime}$ in psychophysics (albeit with dif- 

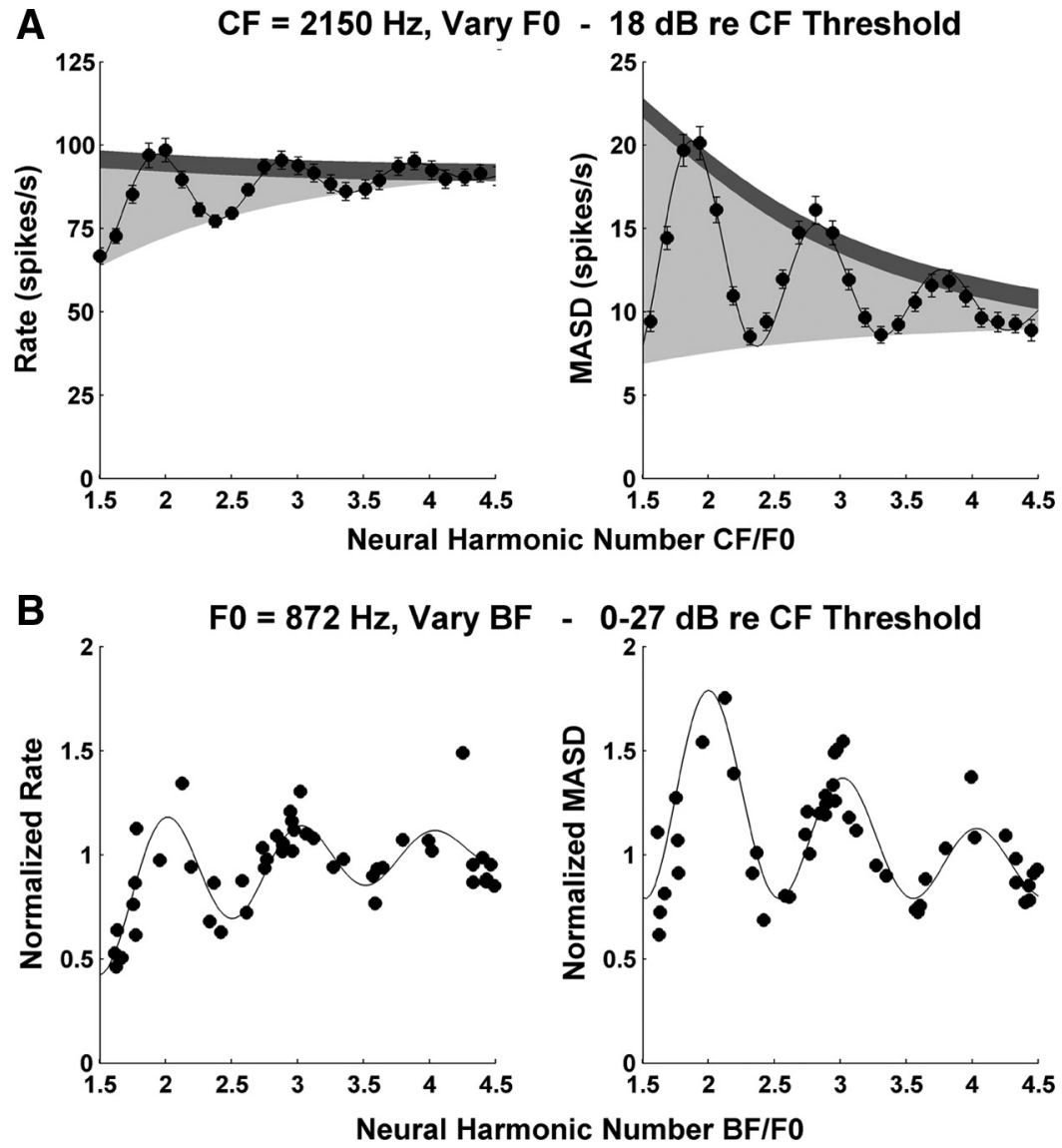

Figure 5. A, Profiles of average rate (left) and MASD (right) against neural harmonic number for an AN fiber ( $\mathrm{CF}, 2150 \mathrm{~Hz}$; same fiber as in middle panel of Fig. 3) in response to a series of harmonic complex tones at $18 \mathrm{~dB}$ re. thresholds. The filled circles show the data points, and the solid lines show best-fitting curves based on Equation 1. The light shadings indicate the area between the top and bottom envelopes of the fitted curve. The dark shadings correspond to two typical SDs of the data points, estimated by bootstrap (see Materials and Methods). The ratio of these two quantities is used as an estimate of the strength of the pitch cues provided by each neural representation. $\boldsymbol{B}$, Interpolation method used for replotting rate and MASD as a function of BF across the AN fiber population for a specific stimulus $F_{0}(872 \mathrm{~Hz})$. Each symbol shows the normalized, interpolated rate (left) or MASD (right) at $F_{0}=872 \mathrm{~Hz}$ for one AN fiber. The solid lines show the best-fitting curves based on Equation 1 . The BF estimated from responses to harmonic complex tones is expressed in dimensionless units $\left(\mathrm{BF} / F_{0}\right)$. Harmonics are in cosine phase. locking to the fine time structure-counteracts the improvement in cochlear frequency selectivity at higher CFs. Above $2700 \mathrm{~Hz}$, the harmonic strength for MASD does not depend statistically on either CF or level, as shown by the horizontal line.

Harmonic strengths for rate and MASD $(<2700 \mathrm{~Hz})$ also differ in their level dependence. For rate, the analysis of covariance shows a significant effect of level group on harmonic strength $(p<$ $0.001)$ and a significant interaction ( $p=$ 0.009) between CF and level, indicating that the degradation in harmonic strength with increasing level is more pronounced for low CFs than for high CFs. The decrease in harmonic strength at high levels may be attributable to the broadening of cochlear filters, rate saturation, or both. In contrast, for MASD, the effect of level on harmonic strength for CFs $<2700 \mathrm{~Hz}$ barely reached statistical significance ( $p=0.033)$. Post hoc paired comparisons (with Bonferroni's corrections) revealed no significant differences in MASD harmonic strengths between any pairs of levels. This result is consistent with our hypothesis that the spatiotemporal representation is more robust with level than the rate representation.

To directly compare the strengths of the rate-place and spatiotemporal representations of resolved harmonics for each fiber, we defined a normalized strength difference as the difference between the harmonic strength of the MASD and that of the rate, divided by their sum. This metric takes values between -1 and +1 , with positive values indicating that the spatiotemporal representation is stronger than the rate-place representation. Figure ferent units) in that it expresses the strength of the "signal" (the oscillation area) in units of SD of the noise. The harmonic strengths for rate and MASD in Figure 5A are 19.6 and 41.0, respectively, consistent with the visual impression that harmonics are better represented in the MASD in this example.

Figure 6 shows harmonic strengths for both average rate (top) and MASD (bottom) plotted against CF for our entire set of responses to complex tones with harmonics in cosine phase. Separate plots are shown for low $(<20 \mathrm{~dB})$, moderate $(20-40 \mathrm{~dB})$, and high $(\geq 40 \mathrm{~dB})$ stimulus levels re. pure-tone thresholds at CF. The solid lines show the linear models (based on analyses of covariance) that best fit the log transformed data across all level groups with the minimum number of free parameters. For the MASD, separate analyses of covariance were run for $\mathrm{CFs}$ below and above $2700 \mathrm{~Hz}$, respectively.

The harmonic strength for rate increases significantly with $\mathrm{CF}(p<0.001)$, consistent with the increase in relative sharpness of cochlear tuning (as expressed by the quality factor $Q$ ) (Kiang et al., 1965; Liberman, 1978). The harmonic strength for MASD also increases with CF $(p<0.001)$, but only up to $2700 \mathrm{~Hz}$, suggesting that another factor-most likely the decrease in phase
7 shows the normalized strength difference against CF for those measurements in which an oscillating curve could be reliably fit to both rate and MASD profiles. As in Figure 6, data are grouped by level relative to the pure-tone threshold at $\mathrm{CF}$ of each fiber. We split the CF range into three groups (with cutoffs at 1350 and $2800 \mathrm{~Hz}$ ) and ran a two-way ANOVA on the normalized strength difference with level and CF groups as factors. There was a significant effect of CF $(p<0.001)$ and a significant interaction between CF and level $(p=0.034)$. We then ran post hoc analyses (with Bonferroni's corrections) to determine whether the mean strength difference in each condition is greater than zero (indicating the spatiotemporal code is better) or smaller than zero (indicating the rate code is better). For CFs $<1350 \mathrm{~Hz}$, the mean strength difference was significantly greater than zero at intermediate and high levels, but not at low levels. For CFs between 1350 and $2800 \mathrm{~Hz}$, the strength difference was only significantly greater than zero at high levels. For CFs $>2800 \mathrm{~Hz}$, the mean strength difference was significantly smaller than zero at all levels. Thus, resolved harmonics are better represented in the MASD profile than in the rate-place profiles at higher stimulus levels, but only for $\mathrm{CFs}<2800 \mathrm{~Hz}$. 
Locations of rate and MASD peaks relative to the fiber $\mathrm{CF}$

Both rate-place and spatiotemporal pitch codes are based on cues to the locations of resolved harmonics via local maxima in response profiles (rate or MASD) along the tonotopic axis. Pitch is putatively extracted by matching these cues to central harmonic templates. The exact locations of these local maxima along the tonotopic axis and their stability with respect to stimulus level are therefore important to the viability (or at least the simplicity) of pitch codes based on these cues. With pure-tone stimuli, the best frequency (BF) of AN fibers (where the firing rate is maximal) is known to be dependent on stimulus level (Rose et al., 1971; Temchin and Ruggero, 2010), and such level dependence is likely to occur with harmonic complex tones as well. The situation is less clear for the MASD, which depends primarily on the phase pattern of the cochlear traveling wave. Although AN fibers with CFs between 1 and $4 \mathrm{kHz}$ often show an inflection point near the CF in their phase-frequency curves for pure-tone stimuli (Palmer and Shackleton, 2009; Temchin and Ruggero, 2010), this inflection seems to be level dependent. In this section, we examine in detail the locations of local maxima in rate and MASD profiles and their level dependence.

A precise estimate of the peak locations in rate and MASD profiles can be obtained from the frequency of the oscillatory component of the fitted curve (i.e., the parameter $\varphi$ in Eq. 1). Specifically, the product of $\varphi$ and the tuning-curve CF gives an estimate of the BF of the fiber based on responses to complex tones. When $\varphi$ equals 1 , the $\mathrm{BF}$ matches the tuning-curve $\mathrm{CF}$ and the oscillations peak exactly at integer values of the neural harmonic number. In the example of Figure $5 A$, local maxima in rate and MASD profiles tend to occur slightly below integer values of the neural harmonic number. As a result, the BFs estimated by fitting both rate and MASD profiles (2210 and $2263 \mathrm{~Hz}$, respectively) are slightly, but significantly higher than the CF estimated from the pure-tone tuning curve $(2150 \mathrm{~Hz})$.

Figure 8 shows the relationships between the $\mathrm{BF}$ estimates derived from rate and MASD profiles and the CF estimate from pure-tone tuning curves for our entire set of responses to complex tones with harmonics in cosine phase. Differences between estimates, expressed as a percentage of the tuning-curve $\mathrm{CF}$, are plotted against the tuning-curve CF. Because a threshold tuning curve is only measured
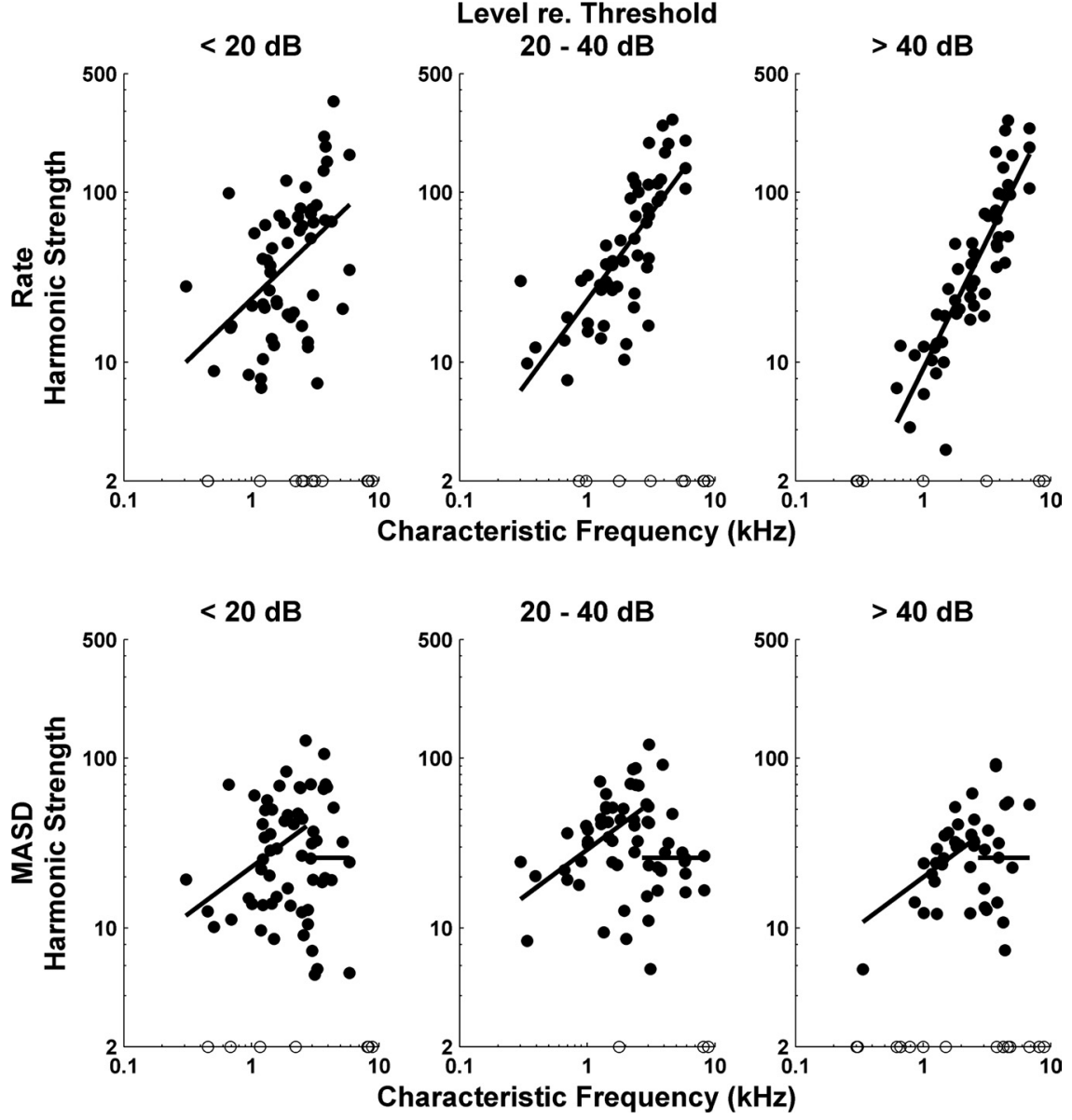

Figure 6. Strength of rate-place and spatiotemporal cues to resolved harmonics as a function of $C F$ for the entire set of responses to complex-tone series with harmonics in cosine phase. The filled circles show harmonic strengths computed from best-fitting curves to profiles of firing rate (top) and MASD (bottom) against neural harmonic number. The open circles along the horizontal axis show data points for which the best-fitting curve had no reliable oscillatory component. Results are grouped by level relative to the threshold of each fiber for a pure tone at CF. The solid lines show best-fitting lines across all level groups obtained from analyses of covariance. Separate analyses were performed for rate and MASD, and, for MASD, for CFs below and above 2700 $\mathrm{Hz}$, respectively.

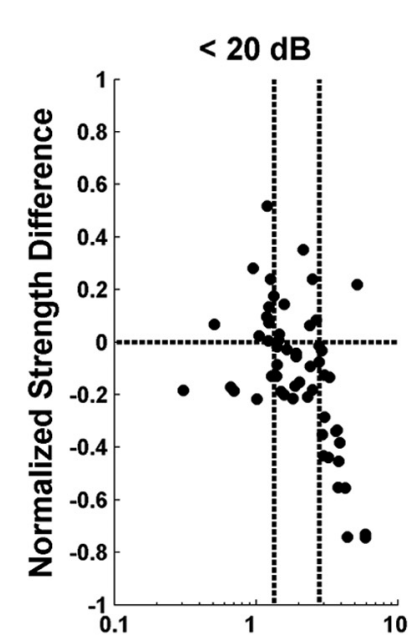

Level re. Threshold
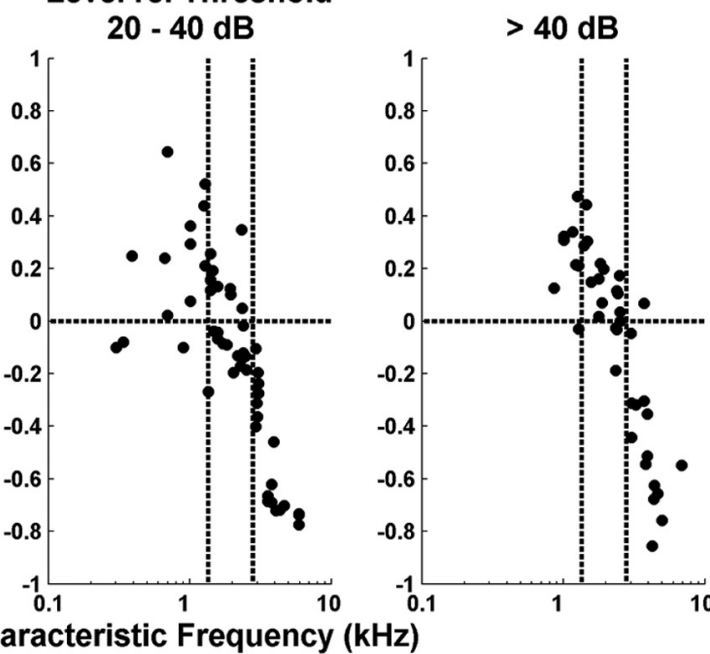

Figure 7. Comparison of the strengths of rate-based and spatiotemporal representations of pitch. Symbols show normalized strength differences as a function of $\mathrm{CF}$ for the AN fiber population. Positive values mean greater strength for the spatiotemporal representation. Results are grouped by level relative to the threshold of each fiber for a pure tone at $C F$ as in Figure 6 . The vertical dashed lines indicate the CF cutoffs used in the ANOVA (see text). Harmonics are in cosine phase. 
A
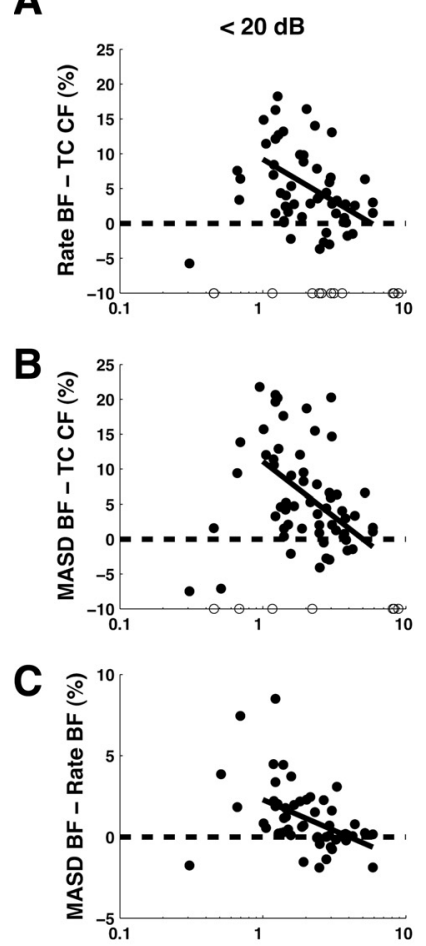

Level re. Threshold
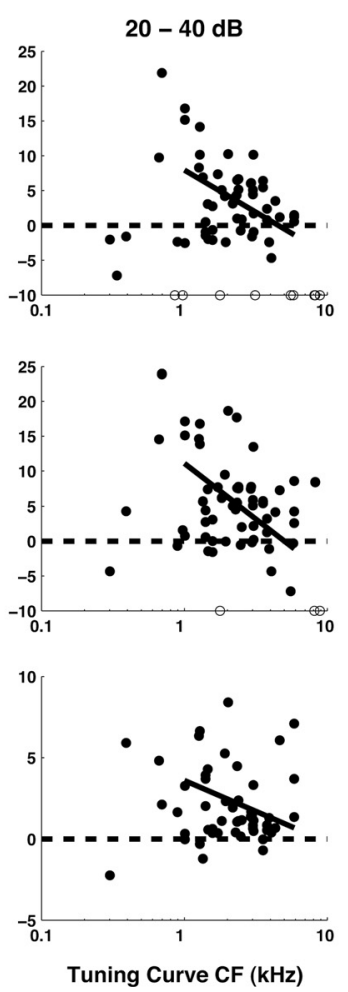
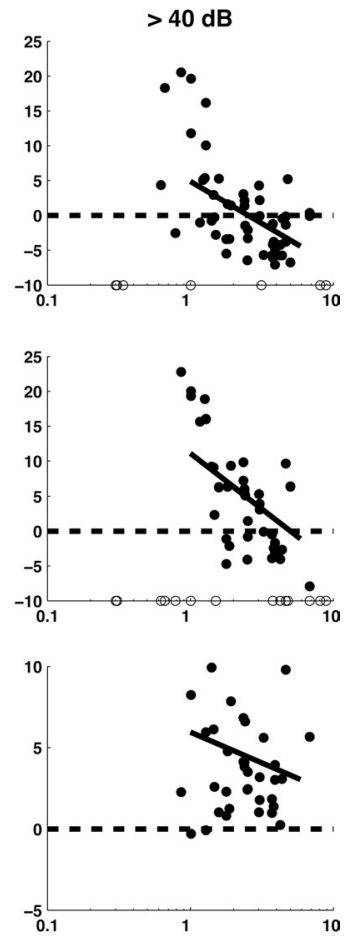

Figure 8. Relationships between BF estimated from rate and MASD profiles in response to harmonic complex tones, and CF estimates from pure-tone tuning curves. The filled circles show differences between estimates, expressed as a percentage of the tuning-curve CF. Results are grouped by stimulus level relative to the threshold of each fiber for a pure tone at CF. The open circles along the horizontal axis indicate measurements for which a BF could not be reliably estimated from rate or MASD profiles. The solid lines show the best-fitting linear models (based on an analysis of covariance) across all levels for $\mathrm{CFs}>1 \mathrm{kHz}$. Harmonics are in cosine phase.

once for each fiber, the tuning-curve CF provides a levelinvariant reference for assessing the level dependence of the BF estimates based on rate and MASD. As in Figure 6, data in Figure 8 are grouped by level relative to the pure-tone threshold at CF of each fiber. For a point to be included, the oscillatory component of the fitted curve (Eq. 1) had to be statistically significant so that the BF could be reliably estimated. The CFs of excluded measurements are indicated by open circles along the horizontal axis in Figure 8. As in Figure 6, the solid lines show the best-fitting linear models across all level groups based on analyses of covariance for CFs $>1 \mathrm{kHz}$.

At low CFs, BFs estimated from both rate (Fig. $8 A$ ) and MASD (Fig. $8 B$ ) tend to be larger than the tuning-curve $\mathrm{CF}$, with differences that can reach $20-25 \%$. Despite considerable scatter in the data, these deviations exhibit a similar decreasing trend with increasing CF in all three level ranges. Overall, the majority of BF estimates based on rate or MASD are larger than the tuning-curve $\mathrm{CFs}$, except for the rate-based $\mathrm{BF}$ at high levels. Importantly, the analyses of covariance show a significant effect of level on BF for rate $(p<0.001)$, but not for MASD $(p>0.01)$, indicating that the BF based on MASD stable with level, whereas the ratebased $\mathrm{BF}$ is not.

MASD-based BF estimates are generally larger than rate-based estimates at all levels (Fig. 8C). An analysis of covariance shows significant effects of both CF $(p=0.008)$ and level $(p<0.001)$, but no interaction. The mean BF difference increases from $0.93 \%$ at low levels to $4.6 \%$ at high levels, but remains substantially smaller than the large deviations observed when comparing ei- ther $\mathrm{BF}$ to the tuning-curve $\mathrm{CF}$ (Fig. $8 A, B)$. These observations are consistent with measurements of basilar membrane velocity in the cochlear base for pure-tone stimuli (Robles and Ruggero, 2001): At a given cochlear place, the frequency for which velocity amplitude is maximum is slightly lower than the frequency that maximizes the group delay (the derivative of the phase with respect to frequency). Moreover, the frequency of maximal group delay appears to be more stable with level than that of maximum amplitude.

The finding that local maxima in the MASD profiles are relatively robust with level may appear to conflict with previous data from AN fibers for either pure tones (Palmer and Shackleton, 2009; Temchin and Ruggero, 2010) or inharmonic complex tones (van der Heijden and Joris, 2006). These studies typically report either plots of phase against stimulus frequency on a linear scale for single fibers, or plots of phase against CF on a logarithmic scale for a sample of AN fibers. When inflection points (corresponding to a local maximum in the phase derivative) are present in these plots, their locations tend to be level dependent, in contrast to MASD maxima in the present study. The MASD is based on a derivative with respect to neural harmonic number $\mathrm{CF} / F_{0}$ to take advantage of cochlear scaling invariance. Phase plots from the previous studies might show more level-invariant inflection points if they were replotted against neural harmonic number rather than linear frequency or log CF. A detailed comparison between these studies is difficult because of differences in stimuli and species [guinea pig for Palmer and Shackleton (2009); chinchilla for Temchin and Ruggero (2010); cat for van der Heijden and Joris (2006) and the present study] and because the MASD does not depend exclusively on phase but also on rate.

Both rate-place and spatiotemporal profiles are labeled line codes in that they require the position along the tonotopic axis to be "known" by higher centers where these profiles are putatively matched to harmonic templates. Traditionally, the label is assumed to be the CF obtained from a pure-tone tuning curve, but this threshold metric may not be appropriate for suprathreshold stimuli. The present results suggest that the BF based on spatiotemporal cues may be a suitable label because it is stable with level and is obtained from responses to harmonic complex tones, which are more common in nature than pure tones.

\section{Phase dependence}

Psychophysical studies show that the pitch value and pitch strength of harmonic complex tones are generally not greatly affected by the phase relationships among the partials so long as the stimuli contain resolved harmonics (Houtsma and Smurzynski, 1990; Carlyon and Shackleton, 1994; Bernstein and Oxenham, 2005). To test whether the spatiotemporal representation is consistent with these perceptual observations, we measured responses to complex tones with harmonics in alternating sine- 

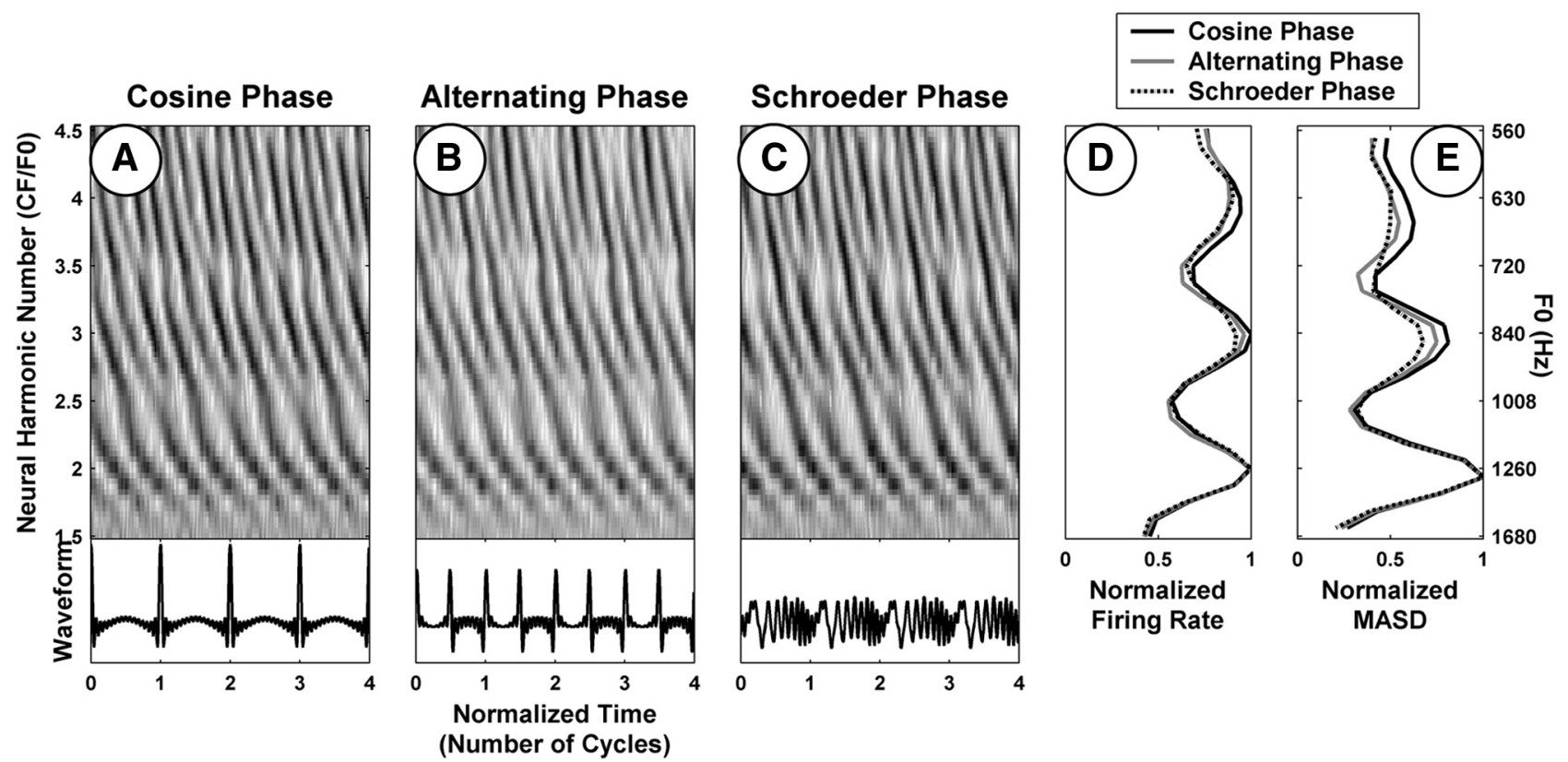

Figure 9. Effect of phase relationship among the harmonics on rate-place and spatiotemporal representations of pitch for one AN fiber (CF, $2530 \mathrm{~Hz}$ ). $\boldsymbol{A}-\boldsymbol{C}$, Pseudo-spatiotemporal response patterns to complex-tone series with harmonics in cosine, alternating (sine- cosine), and Schroeder phase, respectively. The corresponding average rate and MASD profiles, normalized by their respective maxima, are plotted in $\boldsymbol{D}$ and $\boldsymbol{E}$, respectively.

cosine phase and negative Schroeder phase as well as cosine phase. Figure 9 compares the responses to harmonic complexes differing in phase patterns for a fiber with a CF of $2520 \mathrm{~Hz}$. In this example, $F_{0}$ ranged from 560 to $1680 \mathrm{~Hz}$ for all three stimuli. Based on previous results (Cedolin and Delgutte, 2005), we expect low-order harmonics to be well resolved in this $F_{0}$ range, and this expectation is confirmed by the presence of peaks at neural harmonic numbers 2,3 , and 4 in the rate profiles for all three stimuli (Fig. $9 D)$. The pseudo-spatiotemporal response patterns are very similar for all three phase conditions (Fig. $9 A-C$ ), as are the strong cues to resolved harmonics present in the MASD profiles (Fig. 9E).

Figure 10 compares the harmonic strengths of the rate-place (top) and spatiotemporal (bottom) representations across a sample of AN fibers for every pair of phase patterns. The CFs of the fibers included in this sample range from 1 to 3 $\mathrm{kHz}$. Harmonic strengths of the MASD for harmonics in cosine and alternating phase are very similar (A), and both are only slightly larger than the harmonic strengths for harmonics in Schroeder phase (B-C). Similarly, we found no statistically significant difference between rate-based harmonic strengths for any of the three phase configurations (D-F), consistent with previous findings (Cedolin and Delgutte, 2005). These results suggest that the saliences of pitch cues available in both the rate-place and spatiotemporal representations are to a large extent independent of the phase relationship among resolved harmonics, consistent with human psychophysical observations.
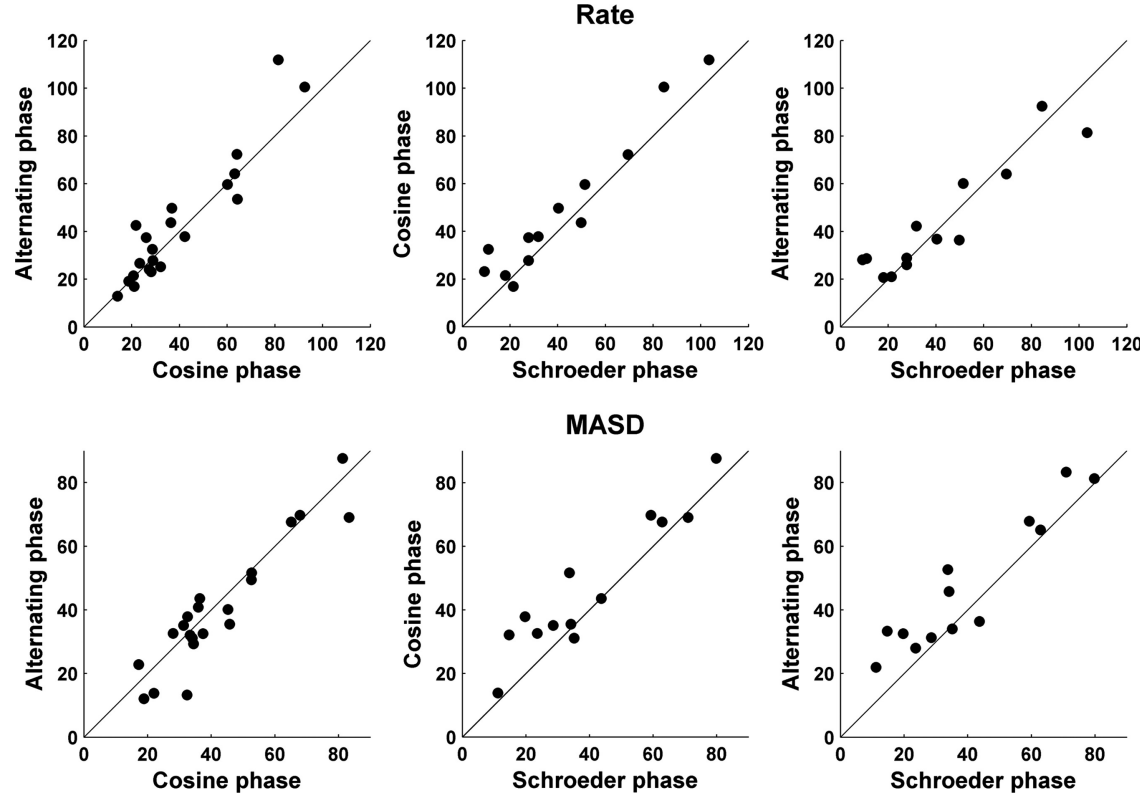

Figure 10. Effect of phase relationships among the harmonics on the strength of the rate-place (top) and spatiotempora (bottom) cues to resolved harmonics for a sample of AN fibers. The filled circles compare harmonic strengths derived from spatiotemporal patterns of response to complex tones with harmonics in cosine, alternating, and Schroeder phase. CFs range from 1 to $3 \mathrm{kHz}$. The solid lines indicate equality.

The phase invariance of the spatiotemporal representation may seem surprising given that this representation strongly depends on the phase pattern of the cochlear traveling wave along the tonotopic axis. However, if a harmonic is well resolved, its local phase pattern (near the place tuned to the harmonic frequency) will be similar to that of a single sinusoid regardless of the phase relationships between this harmonic and neighboring ones. The phase relationship between two neighboring resolved harmonics does affect the spatiotemporal response patterns at 
CFs that lie halfway between these two harmonics (Fig. 9, compare $A, B$, for neural harmonic numbers near 2.5 and 3.5).

\section{Pitch estimation from neural population responses}

So far, we have relied on the assumption of cochlear scaling invariance to interpret profiles of rate and MASD as a function of $\mathrm{CF} / F_{0}$ for single fibers as being equivalent to spatial patterns along the tonotopic axis of the cochlea. We found that the spatiotemporal cues to resolved harmonics are most robust for CFs between 1 and $2.8 \mathrm{kHz}$. For comparison with psychophysical data, this CF range needs to be mapped into a range of stimulus $F_{0}$. For this purpose, we used an interpolation procedure to replot our rate and MASD data as a function of BF for a given stimulus $F_{0}$. Figure $5 B$ illustrates this procedure for an $F_{0}$ of $872 \mathrm{~Hz}$. Because the $F_{0}$ values tested for each fiber are chosen so that the ratio $\mathrm{CF} / F_{0}$ varies from 1.5 to 4.5 in steps of $1 / 8$, few, if any, fibers provide data at this exact $F_{0}$. However, fibers with CFs between $1300 \mathrm{~Hz}(1.5 \times 872)$ and $3900 \mathrm{~Hz}(4.5 \times 872)$ do provide data for $F_{0}$ values close to the desired $872 \mathrm{~Hz}$. For each of these fibers, we used the fitted curves based on Equation 1 (as in Fig. 5A) to interpolate the rate and MASD at the desired $F_{0}(872 \mathrm{~Hz})$. In addition, because average rates and MASD in response to the same stimulus can substantially differ among fibers with similar CFs, we normalized the firing rates (and MASD) of each fiber by their median value over all $F_{0}$ tested.

Figure $5 B$ shows the interpolated, normalized rates and MASD for an $F_{0}$ of $872 \mathrm{~Hz}$ as a function of $\mathrm{BF}$ (expressed as neural harmonic number $\mathrm{BF} / F_{0}$ ) for all the fibers studied with harmonic complex tones in cosine phase at levels $<27 \mathrm{~dB}$ re. threshold. We use the BFs estimated from responses to complex tones rather than the less reliable tuning-curve $\mathrm{CF}$, and we take the geometric mean of the BF estimates for rate and MASD when both are available. The interpolated data show considerably more scatter than typically seen in the single-fiber data (Figs. 3, 4, 5A), reflecting the somewhat ad hoc normalization as well variability resulting from pooling data across a range of levels and across different animals. Despite this scatter, both rate and MASD tend to peak near integer values of the neural harmonic number. The same oscillating curve (Eq. 1) was fit to the interpolated population data as for the single-fiber data. Since this function shows peaks at harmonically related BFs, we are effectively fitting a harmonic template to the profiles of rate and MASD along the tonotopic map in much the same way as in spectral models of pitch (Goldstein, 1973; Wightman, 1973; Cohen et al., 1995). For both rate and MASD, the period of the damped oscillation in the fitted curve (the parameter $1 / \varphi$ in Eq. 1 ) gives an estimate of the stimulus $F_{0}$ from the neural population response. In this case, both $F_{0}$ estimates $(870 \mathrm{~Hz}$ for MASD and $855 \mathrm{~Hz}$ for rate) deviate by $<2 \%$ from the actual $F_{0}(872 \mathrm{~Hz})$. However, since the independent variable for curve fitting is the $\mathrm{BF}$ derived from responses to harmonic complex tones, the $F_{0}$ estimates are expected to be unbiased. More meaningful are the SDs of the $F_{0}$ estimates [computed from the root-mean-square residuals and the Jacobian at the solution vector (Press et al., 1988)], which are a measure of their precision. In the example of Figure $5 B$, the SDs are $\sim 1 \%$ of the actual $F_{0}$.

The analysis of Figure $5 B$ was repeated for $F_{0}$ values ranging from 100 to $1600 \mathrm{~Hz}$ in 1/8-octave steps. Figure 11 shows the SDs of the $F_{0}$ estimates based on rate and MASD profiles as a function of stimulus $F_{0}$. Results are shown separately for two different level ranges, using a cutoff of $27 \mathrm{~dB}$ re. threshold that splits the data in two approximately equal halves. For both metrics and both level ranges, the SDs of the $F_{0}$ estimates are mostly $<1.5 \%$ and show no
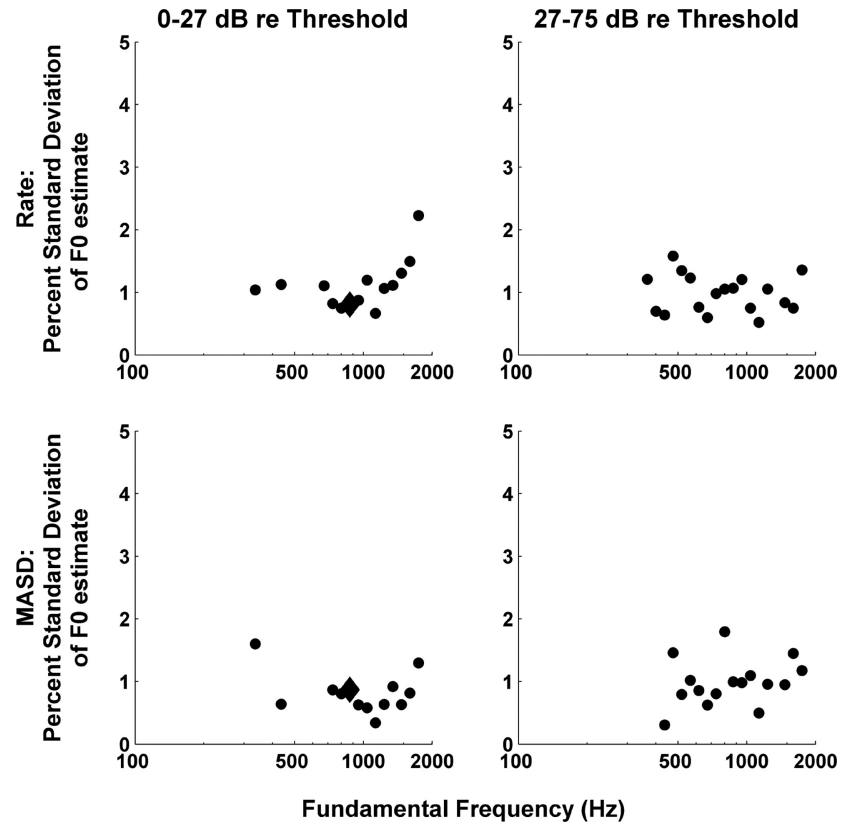

Figure 11. SD of the $F_{0}$ estimated from tonotopic profiles of interpolated rate (top) and MASD (bottom) as a function of stimulus $F_{0}$ for two ranges of stimulus levels. SDs are expressed as a percentage of the actual $F_{0}$ and were computed by fitting Equation 1 to the rate and MASD profiles (as in Fig. 5B) and using the Jacobian of the best-fitting parameter vector.

strong dependence on $F_{0}$. Data are only shown for $F_{0}$ values where an oscillatory curve could be reliably fit to the interpolated rate and MASD profiles (as assessed by an $F$ test) (see Materials and Methods). For some $F_{0}$ values, the sampling of BFs was sparse and/or oddly distributed, making $F_{0}$ estimation unreliable. Such unpredictable sampling of the tonotopic axis is the very reason why we adopted an experimental design based on cochlear scaling invariance, which ensures a fine, uniform sampling of neural harmonic numbers for all fibers.

The lowest $F_{0}$ for which a clear oscillatory component could be detected in either rate or MASD profiles was $336 \mathrm{~Hz}$ at lower levels, and $367 \mathrm{~Hz}$ at higher levels. It is harder to define an upper limit for $F_{0}$ estimation based on MASD. At the higher levels, MASD-based $F_{0}$ estimation tended to be less reliable than ratebased estimation for $F_{0}$ values $>1100 \mathrm{~Hz}$, as expected from the degradation in phase locking. However, at the lower levels, $F_{0}$ could be estimated from MASD profiles up to the highest value tested $(1600 \mathrm{~Hz})$. The MASD is likely to be overestimated at high frequencies because of intrinsic noise in neural firings. The spatial derivative operation amplifies high-frequency noise in temporal response patterns. This noise is not attenuated at the subsequent temporal integration stage because it is the absolute value of the spatial derivative that is integrated. If the noise is Poisson-like, as expected for AN fibers, then the higher the firing rate, the higher the noise. Thus, the oscillations in MASD profiles at high $F_{0}$ values may simply be noise modulations directly resulting from the observed oscillations in firing rate. Despite this difficulty, $F_{0}$ estimation from MASD profiles was overall most effective for $F_{0}$ values between $\sim 350$ and $1100 \mathrm{~Hz}$.

\section{Discussion}

\section{Spatiotemporal representation of pitch}

We tested the effectiveness of a spatiotemporal representation of the pitch of harmonic complex tones in the cat auditory nerve. We found that strong spatiotemporal cues to resolved harmonics are available in the responses of AN fibers whose CFs are high 
enough for harmonics to be sufficiently resolved $(>1 \mathrm{kHz})$ but below the limit $(\sim 2.8 \mathrm{kHz})$ above which phase locking is significantly degraded. For fibers with $\mathrm{CF}<1 \mathrm{kHz}$, rate-place cues to resolved harmonics are also weak because of poor harmonic resolvability, consistent with previous studies (Sachs and Young, 1979; Cedolin and Delgutte, 2005). At high CFs, however, the rate-place representation improves because of the progressive sharpening of cochlear tuning relative to the CF (Kiang et al., 1965; Shera et al., 2002; Cedolin and Delgutte, 2005), whereas the spatiotemporal representation degrades because of the decline in phase locking (Johnson, 1980). In the CF range in which it is effective, the spatiotemporal representation is more robust than the rate-place representation at high stimulus levels. Whereas the effectiveness of the rate-pace representation is limited by firing rate saturation (Sachs and Young, 1979), phase locking remains robust at levels at which rate is saturated (Young and Sachs, 1979). Nevertheless, the spatiotemporal representation does degrade somewhat at high levels consistent with broader cochlear tuning and associated flattening in the frequency dependence of the phase response (Anderson et al., 1971; Palmer and Shackleton, 2009; Temchin and Ruggero, 2010).

Our primary results are based on the assumption of local scaling invariance in cochlear mechanics (Zweig, 1976), which holds only approximately (Shera and Guinan, 2003; van der Heijden and Joris, 2006). Although scaling invariance implies cochlear filters with constant $Q$ (the ratio of $C F$ to bandwidth), $Q$ is actually an increasing power function of CF in cat AN (Shera et al., 2002; Shera and Guinan, 2003; Cedolin and Delgutte, 2005). This power law (with an exponent of $\sim 0.37$ ) predicts that $Q$ varies by no more than $\pm 21 \%$ over the 1.6 octave range of $C F / F_{0}$ spanned by our stimuli. Although not insignificant, these deviations are probably within the range of experimental error. Scaling invariance further prescribes that all temporal parameters of cochlear processing scale with $\mathrm{CF}$, whereas some of these parameters such as the upper frequency limit of phase locking clearly do not (Johnson, 1980). Using model simulations, Larsen et al. (2008) found that assuming scaling invariance tends to overestimate phase locking to higher harmonics (relative to the mean neural harmonic number 3), and underestimate phase locking to lower harmonics. This effect can be discerned in the model results of Figure 2, in which the decay of the MASD with increasing neural harmonic number is somewhat less pronounced in the right panel (in which scaling invariance is assumed) than in the left panel (in which it is not).

Our interpolation procedure for replotting rate and MASD as a function of $\mathrm{BF}$ for a given $F_{0}$ (Fig. $5 B$ ) bears on the issue of scaling invariance because, at least for rate, it eliminates the constant $Q$ assumption. For the interpolated data, the spatiotemporal representation worked best for $F_{0}$ values between 350 and $1100 \mathrm{~Hz}$. Within that range, the stimulus $F_{0}$ could be estimated with a $\mathrm{SD}<2 \%$ based on data from only 30-50 AN fibers. These precision figures are only indicative, as they depend on experimental variables such as the number of fibers sampled and the distribution of CFs in the sample.

The $F_{0}$ range over which the spatiotemporal representation of pitch is most effective in cat AN encompasses the $500-1000 \mathrm{~Hz}$ range of cat vocalizations (Brown et al., 1978; Shipley et al., 1991). What might be the corresponding range in humans? Evidence from both otoacoustic emissions and psychophysics suggests that cochlear frequency resolution may be up to three times finer in humans than in cats (Shera et al., 2002; Oxenham and Shera, 2003; Shera et al., 2010) [but see Ruggero and Temchin (2005) for a contrary opinion]. If so, the lower $F_{0}$ limit for a viable spatio- temporal representation of pitch would be $\sim 120 \mathrm{~Hz}$ in humans. The upper limit at $\sim 1100 \mathrm{~Hz}$ would remain the same providing the frequency dependence of phase locking is similar in cats and humans. This $F_{0}$ range encompasses most of the range of spoken human voice $(80-350 \mathrm{~Hz})$, and the upper limit is approximately consistent with the $\sim 1300 \mathrm{~Hz}$ upper limit of pitch perception for missing-fundamental stimuli containing many harmonics (Moore, 1973).

\section{Neural codes for pitch}

The spatiotemporal representation of pitch offers a number of advantages over traditional codes for the pitch of complex tones. It is more robust with stimulus level than the rate-place representation and, unlike the rate-place representation, predicts an upper frequency limit to the pitch of missing-fundamental stimuli approximately consistent with psychophysical data. Although the autocorrelation (also known as interspike interval) model of pitch has trouble predicting the stronger pitch produced by stimuli containing resolved harmonics compared with stimuli consisting entirely of unresolved harmonics (Carlyon and Shackleton, 1994; Carlyon, 1998; Bernstein and Oxenham, 2005; Cedolin and Delgutte, 2005), the spatiotemporal representation intrinsically requires resolved harmonics. The autocorrelation model also has trouble explaining the poor temporal pitch perception with cochlear implants (Shannon, 1983; Townshend et al., 1987) because phase locking of AN fibers is excellent with electric stimulation of the cochlea (Dynes and Delgutte, 1992; Shepherd and Javel, 1997). In contrast, the lack of a cochlear traveling wave with cochlear implants precludes the phase cues on which the spatiotemporal representation is based. Nevertheless, if pitch perception with resolved harmonics were based primarily on spatiotemporal cues, a second pitch mechanism would be required to account for the weak pitch produced by unresolved harmonics and stimulation through cochlear implants (de Cheveigné, 2005).

The pitch representation tested in this paper (Shamma, 1985) is called "spatiotemporal" because it makes use of both the spatial distribution of AN activity along the tonotopic axis and the precise phase locking of spikes to the fine time structure of the stimulus waveform. Other spatiotemporal models of auditory processing (Loeb et al., 1983; Shamma and Klein, 2000; Carney and Heinz, 2002) also critically depend on the relative timing of spikes from different cochlear locations and rely on the delays created by the cochlear traveling wave rather than postulating neural delay lines as in the autocorrelation model. Nevertheless, these spatiotemporal models differ in important ways. All three models just cited differ from the present one in that they use cross-correlation (or equivalently coincidence detection) rather than lateral inhibition to compare the timing of spikes across cochlear locations. The models of both Loeb et al. (1983) and Shamma and Klein (2000) involve cross-correlation over much wider extents of the tonotopic axis (CF separations of up to several octaves) than the local comparisons used in the present model. The wide tonotopic span of spatial correlations in the model of Shamma and Klein (2000) is consistent with its purpose of explaining the formation of harmonic templates, and this model is compatible with the spatial derivative as a front end. Although level-dependent changes in cochlear tuning play a critical role in the phase opponency model of Carney et al. (2002), the phase cues used by the present model directly follow from linear systems theory (Goldstein et al., 1971). Apparently, the cochlear nonlinearities are mild enough that 
the spatiotemporal phase cues are fairly robust over the level range tested.

In summary, although several models combining place and temporal information have been proposed for pitch and other perceptual phenomena, these models are clearly distinct, and each has to be evaluated on its own merit. The present study represents the first detailed physiological evaluation of a spatiotemporal pitch model.

\section{Neural mechanisms for extracting spatiotemporal pitch cues}

A key question is whether the spatiotemporal cues to resolved harmonics available in the patterns of AN activity are actually extracted in the CNS. A neural mechanism that extracts spatiotemporal cues must be sensitive to the relative timing of spikes from AN fibers innervating neighboring cochlear locations. Because phase locking to the waveform fine structure rapidly degrades in the ascending auditory pathway (Langner, 1992), an early brainstem processing site such as the cochlear nucleus or, perhaps, the medial superior olive (Loeb et al., 1983) is a logical place for such a mechanism.

The simplest possibility is a lateral inhibitory mechanism, as originally proposed by Shamma (1985) and implemented here via the MASD. There is evidence for lateral inhibition in the dorsal cochlear nucleus (DCN), where principal cells receive both excitatory input from AN fibers and a lower-CF inhibitory input from vertical cells (Voigt and Young, 1990). However, phase locking to the fine structure is generally poor in DCN cells (Lavine, 1971; Rhode and Smith, 1986), so these neurons seem ill suited for the fast temporal processing required to decode spatiotemporal cues. Nevertheless, neurons might perform the required computations in their dendrites even if they have poor phase locking because of subsequent low-pass filtering. Although bushy cells in the ventral cochlear nucleus (VCN) do show good phase locking, the BF of inhibition in these neurons appears to match the excitatory BF (i.e., the inhibition is not lateral) (Caspary et al., 1994; Kopp-Scheinpflug et al., 2002). However, these experiments may not have been sufficiently sensitive to detect small BF differences, so that a role for lateral inhibition in processing spatiotemporal cues cannot be ruled out.

An alternative decoding mechanism for spatiotemporal cues is across-CF coincidence detection (Carney, 1994; Carney and Heinz, 2002) for which there is evidence in some phase-locking VCN units (Carney, 1990; Joris et al., 1994; Wang and Delgutte, 2009). A potential difficulty is that fewer spike coincidences are expected at the cochlear locations tuned to resolved harmonics (i.e., rate and phase cues tend to oppose each other, whereas they act in synergy in a lateral inhibitory circuit). Thus, a coincidence detection mechanism may work best at higher stimulus levels at which the rate is saturated.

\section{Conclusion}

We tested a spatiotemporal representation of pitch based on phase cues to resolved harmonics that are created by the cochlear traveling wave and can, in principle, be extracted by a lateralinhibition mechanism. This representation is effective over a range of $F_{0}$ values whose lower limit is determined by the frequency selectivity of the cochlea, whereas the upper limit is caused by the degradation in phase locking. In cats, the spatiotemporal representation is viable over the $F_{0}$ range of cat vocalizations, and this correspondence may also approximately hold in humans because of the putatively sharper cochlear frequency tuning in that species. The spatiotemporal representation is con- sistent with key trends in pitch psychophysics and is more robust than the rate-place representation at high stimulus levels.

\section{References}

Anderson DJ, Rose JE, Hind JE, Brugge JF (1971) Temporal position of discharges in single auditory nerve fibers within the cycle of a sine-wave stimulus: frequency and intensity effects. J Acoust Soc Am 49 [Suppl 2]:1131-1154.

Bendor D, Wang X (2005) The neuronal representation of pitch in primate auditory cortex. Nature 436:1161-1165.

Bernstein JG, Oxenham AJ (2005) An autocorrelation model with place dependence to account for the effect of harmonic number on fundamental frequency discrimination. J Acoust Soc Am 117:3816-3831.

Brown KA, Buchwald JS, Johnson JR, Mikolich DJ (1978) Vocalization in the cat and kitten. Dev Psychobiol 11:559-570.

Cariani PA, Delgutte B (1996a) Neural correlates of the pitch of complex tones. I. Pitch and pitch salience. J Neurophysiol 76:1698-1716.

Cariani PA, Delgutte B (1996b) Neural correlates of the pitch of complex tones. II. Pitch shift, pitch ambiguity, phase invariance, pitch circularity, rate pitch, and the dominance region for pitch. J Neurophysiol 76:1717-1734.

Carlyon RP (1998) Comments on "A unitary model of pitch perception" [J. Acoust. Soc. Am. 102, 1811-1820 (1997)]. J Acoust Soc Am 104:1118-1121.

Carlyon RP, Shackleton TM (1994) Comparing the fundamental frequencies of resolved and unresolved harmonics: evidence for two pitch mechanisms? J Acoust Soc Am 95:3541-3554.

Carney LH (1990) Sensitivities of cells in the anteroventral cochlear nucleus of cat to spatiotemporal discharge patterns across primary afferents. J Neurophysiol 64:437-456.

Carney LH (1994) Spatiotemporal encoding of sound level: models for normal encoding and recruitment of loudness. Hear Res 76:31-44.

Carney LH, Heinz MG (2002) Auditory phase opponency: a temporal model for masked detection at low frequencies. Acta Acust 88:334-346.

Carney LH, Yin TC (1988) Temporal coding of resonances by lowfrequency auditory nerve fibers: single-fiber responses and a population model. J Neurophysiol 60:1653-1677.

Caspary DM, Backoff PM, Finlayson PG, Palombi PS (1994) Inhibitory inputs modulate discharge rate within frecuency receptive fields of anteroventral cochlear nucleus neurons. J Neurophysiol 72:2124-2133.

Cedolin L, Delgutte B (2005) Pitch of complex tones: rate-place and interspike interval representations in the auditory nerve. J Neurophysiol 94:347-362.

Cohen MA, Grossberg S, Wyse LL (1995) A spectral network model of pitch perception. J Acoust Soc Am 98:862-879.

de Cheveigné A (2005) Pitch perception models. In: Pitch: neural coding and perception (Plack CJ, Oxenham AJ, Fay RR, Popper AN, eds), pp 169-233. New York: Springer.

Dynes SB, Delgutte B (1992) Phase-locking of auditory-nerve discharges to sinusoidal electric stimulation of the cochlea. Hear Res 58:79-90.

Efron B, Tibshirani RJ (1993) An introduction to the bootstrap. New York: Chapman and Hall.

Evans EF (1983) Pitch and cochlear nerve fibre temporal discharge patterns. In: Hearing: physiological bases and psychophysics (Klinke R, Hartmann R, eds), pp 140-146. Berlin: Springer.

Goldstein JL (1973) An optimum processor theory for the central formation of the pitch of complex tones. J Acoust Soc Am 54:1496-1516.

Goldstein JL, Baer T, Kiang NYS (1971) A theoretical treatment of latency, group delay, and tuning characteristics for auditory-nerve responses to clicks and tones. In: Physiology of the auditory system (Sachs MB, ed), pp 133-141. Baltimore: National Educational Consultants.

Houtsma AJM, Smurzynski J (1990) Pitch identification and discrimination for complex tones with many harmonics. J Acoust Soc Am 87:304-310.

Javel E (1980) Coding of AM tones in the chinchilla auditory nerve: implications for the pitch of complex tones. J Acoust Soc Am 68:133-146.

Johnson DH (1980) The relationship between spike rate and synchrony in responses of auditory-nerve fibers to single tones. J Acoust Soc Am 68:1115-1122.

Joris PX, Carney LH, Smith PH, Yin TC (1994) Enhancement of neural synchronization in the anteroventral cochlear nucleus. I. Responses to tones at the characteristic frequency. J Neurophysiol 71:1022-1036.

Kiang NY, Moxon EC (1974) Tails of tuning curves of auditory-nerve fibers. J Acoust Soc Am 55:620-630. 
Kiang NYS, Watanabe T, Thomas EC, Clark LF (1965) Discharge patterns of single fibers in the cat's auditory nerve. Cambridge, MA: MIT.

Kopp-Scheinpflug C, Dehmel S, Dörrscheidt GJ, Rübsamen R (2002) Interaction of excitation and inhibition in anteroventral cochlear nucleus neurons that receive large endbulb synaptic endings. J Neurosci 22:11004-11018.

Langner G (1992) Periodicity coding in the auditory system. Hear Res 60:115-142.

Larsen E, Cedolin L, Delgutte B (2008) Pitch representations in the auditory nerve: two concurrent complex tones. J Neurophysiol 100:1301-1319.

Lavine RA (1971) Phase-locking in response of single neurons in cochlear nuclear complex of the cat to low frequency tonal stimuli. J Neurophysiol 34:467-483.

Liberman MC (1978) Auditory-nerve responses from cats raised in a lownoise chamber. J Acoust Soc Am 63:442-455.

Licklider JC (1951) A duplex theory of pitch perception. Experientia $7: 128-134$.

Loeb GE, White MW, Merzenich MM (1983) Spatial cross-correlation. A proposed mechanism for acoustic pitch perception. Biol Cybern 47:149-163.

Meddis R, Hewitt MJ (1991a) Virtual pitch and phase sensitivity of a computer model of the auditory periphery. I. Pitch identification. J Acoust Soc Am 89:2866-2882.

Meddis R, Hewitt MJ (1991b) Virtual pitch and phase sensitivity of a computer model of the auditory periphery. II. Phase sensitivity. J Acoust Soc Am 89:2883-2894.

Moore BC (1973) Some experiments relating to the perception of complex tones. Q J Exp Psychol 25:451-475.

Oxenham AJ, Shera CA (2003) Estimates of human cochlear tuning at low levels using forward and simultaneous masking. J Assoc Res Otolaryngol 4:541-554.

Palmer AR (1990) The representation of the spectra and fundamental frequencies of steady-state single- and double-vowel sounds in the temporal discharge patterns of guinea pig cochlear-nerve fibers. J Acoust Soc Am 88:1412-1426.

Palmer AR, Shackleton TM (2009) Variation in the phase of response to low-frequency pure tones in the guinea pig auditory nerve as functions of stimulus level and frequency. J Assoc Res Otolaryngol 10:233-250.

Pfeiffer RR, Kim DO (1975) Cochlear nerve fiber responses: distribution along the cochlear partition. J Acoust Soc Am 58:867-869.

Press WH, Flannery BP, Teukolsky SA, Vetterling WT (1988) Numerical recipes in C. The art of scientific computing. Cambridge, UK: Cambridge UP.

Rhode WS (1995) Interspike intervals as a correlate of periodicity pitch in cat cochlear nucleus. J Acoust Soc Am 97:2413-2429.

Rhode WS, Smith PH (1986) Physiological studies on neurons in the dorsal cochlear nucleus of cat. J Neurophysiol 56:287-307.

Robles L, Ruggero MA (2001) Mechanics of the mammalian cochlea. Physiol Rev 81:1305-1352.

Rose JE, Hind JE, Anderson DJ, Brugge JF (1971) Some effects of stimulus intensity on response of auditory nerve fibers in the squirrel monkey. J Neurophysiol 34:685-699.

Ruggero MA, Temchin AN (2005) Unexceptional sharpness of frequency tuning in the human cochlea. Proc Natl Acad Sci U S A 102:18614-18619.

Sachs MB, Young ED (1979) Encoding of steady-state vowels in the auditory nerve: representation in terms of discharge rate. J Acoust Soc Am 66:470-479.

Sayles M, Winter IM (2008) Ambiguous pitch and the temporal representation of inharmonic iterated rippled noise in the ventral cochlear nucleus. J Neurosci 28:11925-11938.
Schouten JF, Ritsma RJ, Cardozo BL (1962) Pitch of the residue. J Acoust Soc Am 34:1418-1424.

Schroeder MR (1970) Synthesis of low peak-factor signals and binary sequences with low autocorrelation. IEEE Trans Inf Theory 16:85-89.

Shamma SA (1985) Speech processing in the auditory system. II: Lateral inhibition and the central processing of speech evoked activity in the auditory nerve. J Acoust Soc Am 78:1622-1632.

Shamma S, Klein D (2000) The case of the missing pitch templates: how harmonic templates emerge in the early auditory system. J Acoust Soc Am 107:2631-2644.

Shannon RV (1983) Multichannel electrical stimulation of the auditory nerve in man. I. Basic psychophysics. Hear Res 11:157-189.

Shepherd RK, Javel E (1997) Electrical stimulation of the auditory nerve. I. Correlation of physiological responses with cochlear status. Hear Res 108:112-144

Shera CA, Guinan JJ Jr (2003) Stimulus-frequency-emission group delay: a test of coherent reflection filtering and a window on cochlear tuning. J Acoust Soc Am 113:2762-2772.

Shera CA, Guinan JJ Jr, Oxenham AJ (2002) Revised estimates of human cochlear tuning from otoacoustic and behavioral measurements. Proc Natl Acad Sci U S A 99:3318-3323.

Shera CA, Guinan JJ Jr, Oxenham AJ (2010) Otoacoustic estimation of cochlear tuning: validation in the chinchilla. J Assoc Res Otolaryngol 11: 343-365.

Shipley C, Carterette EC, Buchwald JS (1991) The effect of articulation on the acoustical structure of feline vocalizations. J Acoust Soc Am 89:902-909.

Shofner WP (1991) Temporal representation of rippled noise in the anteroventral cochlear nucleus of the chinchilla. J Acoust Soc Am 90:2450-2466.

Shofner WP (1999) Responses of cochlear nucleus units in the chinchilla to iterated rippled noises: analysis of neural autocorrelograms. J Neurophysiol 81:2662-2674.

Temchin AN, Ruggero MA (2010) Phase-locked responses to tones of chinchilla auditory nerve fibers: implications for apical cochlear mechanics. J Assoc Res Otolaryngol 11:297-318.

Townshend B, Cotter N, Van Compernolle D, White RL (1987) Pitch perception by cochlear implant subjects. J Acoust Soc Am 82:106-115.

van der Heijden M, Joris PX (2006) Panoramic measurements of the apex of the cochlea. J Neurosci 26:11462-11473.

Voigt HF, Young ED (1990) Cross-correlation analysis of inhibitory interactions in dorsal cochlear nucleus. J Neurophysiol 64:1590-1610.

Wang GI, Delgutte B (2009) Spatio-temporal processing of auditory-nerve activity in the cochlear nucleus. Abstr Assoc Res Otolaryngol 32:845.

Wiegrebe L, Winter IM (2001) Temporal representation of iterated rippled noise as a function of delay and sound level in the ventral cochlear nucleus. J Neurophysiol 85:1206-1219.

Wightman FL (1973) The pattern-transformation model of pitch. J Acoust Soc Am 54:407-416.

Winter IM, Wiegrebe L, Patterson RD (2001) The temporal representation of the delay of iterated rippled noise in the ventral cochlear nucleus of the guinea-pig. J Physiol 537:553-566.

Young ED, Sachs MB (1979) Representation of steady-state vowels in the temporal aspects of the discharge patterns of populations of auditorynerve fibers. J Acoust Soc Am 66:1381-1403.

Zhang X, Heinz MG, Bruce IC, Carney LH (2001) A phenomenological model for the responses of auditory-nerve fibers: I. Nonlinear tuning with compression and suppression. J Acoust Soc Am 109:648-670.

Zweig G (1976) Basilar membrane motion. Cold Spring Harbor Symp Quant Biol 40:619-633. 